\title{
COMPARISON OF MOMENT METHOD AND FOURIER ANALYSIS IN CHROMATOGRAPH*
}

\author{
Noriaki WAKAO AND KenjI TANAKA \\ Department of Chemical Engineering, Yokohama National \\ University, Yokohama, Japan
}

\begin{abstract}
Analytical solution was obtained for response to delta tracer signal which is injected into a packed bed of non-adsorbing porous particles. Moment method and Fourier analysis were compared in estimating parameters from delta response with truncation error in tail.
\end{abstract}

Since $\mathrm{Kubin}^{8)}$ and Kucera ${ }^{9)}$ proposed a moment method for evaluating parameters of packed-bed reactors, much attention has been focused on chromatographic parameter estimation techniques.

In experiments Smith et al. ${ }^{4,11,12)}$ have applied the moment method to several cases. Some other methods have also been proposed: weighted moment $\operatorname{method}^{10)}$, transfer function fitting $\operatorname{method}^{7,10}$ and Fourier transform fitting method $^{5)}$.

Anderssen and White ${ }^{2}$ compared these estimation methods using experimental signals for a packed bed of non-porous particles and have concluded that the best method was time domain fitting, and that the Fourier transform fitting and the weighted moment methods also gave quite accurate values. A similar conclusion has been reached by Clements ${ }^{5)}$ and Böxkes and Hofmann ${ }^{3)}$.

Gangwall, Hudgins, Bryson and Silveston ${ }^{6}$. have demonstrated that Fourier analysis was sufficiently usable.

The present paper concerns a packed bed of nonadsorbing porous particles. The purposes of this investigation are, first, to obtain an analytical solution for delta response and, second, to compare the moment method and Fourier analysis in illustrating the influence of truncation errors in tailing portion on the estimated parameter values.

\section{Analytical Solution}

Let us consider a case where a non-adsorbable tracer pulse signal is imposed on a steadily flowing fluid in a packed bed of porous spherical particles of uniform size. The differential equation for the bulk concentration is

$$
\frac{\partial C}{\partial t}=D_{a x} \frac{\partial^{2} C}{\partial X^{2}}-U \frac{\partial C}{\partial X}-\frac{3 D_{e}}{R} \frac{1-\varepsilon_{b}}{\varepsilon_{b}}\left(\frac{\partial c_{i}}{\partial r}\right)_{r=R}
$$

The concentration in the particle is

$$
\frac{\partial c_{i}}{\partial t}=\frac{D_{e}}{\varepsilon_{p}}\left(\frac{\partial^{2} c_{i}}{\partial r^{2}}+\frac{2}{r} \frac{\partial c_{i}}{\partial r}\right)
$$

with

$$
\begin{aligned}
& C=0, \quad \text { at } \quad t=0 \\
& c_{i}=0, \quad \text { at } t=0 \\
& D_{e} \frac{\partial c_{i}}{\partial r}=k_{f}\left(C-c_{i}\right), \text { at } r=R \\
& C=\delta(t=0), \quad \text { at } \quad X=0 \\
& \frac{\partial C}{\partial X}=0, \quad \text { at } \quad X=L
\end{aligned}
$$

The system of Eqs. (1) to (7) may be made dimensionless :

$$
\begin{aligned}
& \frac{\partial C}{\partial \tau}=\beta \frac{\partial^{2} C}{\partial x^{2}}-\frac{\partial C}{\partial x}-\frac{3 \eta}{\kappa}\left(\frac{\partial c_{i}}{\partial \rho}\right)_{\rho=1} \\
& \kappa \frac{\partial c_{i}}{\partial \tau}=\frac{\partial^{2} c_{i}}{\partial \rho^{2}}+\frac{2}{\rho} \frac{\partial c_{i}}{\partial \rho} \\
& C(x, 0)=0 \\
& c_{i}(\rho, x, 0)=0 \\
& \gamma\left(\frac{\partial c_{i}}{\partial \rho}\right)_{\rho=1}=C-\left(c_{i}\right)_{\rho=1} \\
& C(0, \tau)=\delta(\tau=0) \\
& \left(\frac{\partial C}{\partial x}\right)_{x=1}=0
\end{aligned}
$$

The tracer concentration in the effluent fluid is (Appendix A)

$$
C_{e}=2 \beta \sum_{n=1}^{\infty} \sum_{j=1}^{\infty} \frac{\alpha_{n} \sin \alpha_{n}}{1-\frac{\sin 2 \alpha_{n}}{2 \alpha_{n}}}
$$




$$
\times \frac{(1-\gamma) \sin z_{j}+\gamma z_{j} \cos z_{j}}{A_{n, j} \sin z_{j}+B_{n, j} \cos z_{j}} e^{-\left(2 \beta z_{j}^{2} \tau-x\right) /(2 \kappa \beta)}
$$

where

$$
\begin{aligned}
& A_{n, j}=1-\gamma+\frac{3 \eta}{2}+\frac{\kappa \gamma}{2}\left(\frac{1}{4 \beta}+\beta \alpha_{n}^{2}-\frac{z_{j}^{2}}{\kappa}\right) \\
& B_{n, j}=\gamma z_{j}-\frac{\kappa}{2 z_{j}}\left(\frac{1}{4 \beta}+\beta \alpha_{n}^{2}-\frac{z_{j}^{2}}{\kappa}\right)
\end{aligned}
$$

$\alpha_{n}$ is a root of

$$
\tan \alpha=-2 \beta \alpha
$$

and $z_{j}$ is a root of

$$
\tan z=-z \frac{\gamma\left(\frac{1}{4 \beta}+\beta \alpha_{n}^{2}-\frac{z^{2}}{\kappa}\right)+\frac{3 \eta}{\kappa}}{(1-\gamma)\left(\frac{1}{4 \beta}+\beta \alpha_{n}^{2}-\frac{z^{2}}{\kappa}\right)-\frac{3 \eta}{\kappa}}
$$

Eq.(15) is a function of $\beta, \gamma, \eta, \kappa$ and $\tau$ or $\varepsilon_{p}, \varepsilon_{b}$, $L / D_{p}, D_{e} / D_{v}, N_{S c} N_{R e}, N_{P e}, N_{S h}$ and $U t / L$. Assuming the following data

$$
\begin{aligned}
\varepsilon_{p} & =0.5, & \varepsilon_{b} & =0.4, \\
L / D_{p} & =10, & N_{S c} N_{R e} & =10,
\end{aligned}
$$

Eq.(15) is illustrated for several combinations of $D_{e} / D_{v}, N_{P e}$ and $N_{S h}$ values in Fig. 1.

\section{Moments}

The Laplace transform, $\bar{C}_{e}(s)$, of the response, $C_{e}$,

$$
\bar{C}_{e}(s)=\int_{0}^{\infty} C_{e} e^{-s \tau} d \tau
$$

may be obtained by applying the transform to the system of Eqs. (8) to (14) and solving them:

$$
\overline{C_{e}}(s)=\frac{2 \psi e^{1 /(2 \beta)}}{\left(\frac{1}{2 \beta}+\psi\right) e^{\phi}-\left(\frac{1}{2 \beta}-\psi\right) e^{-\psi}}
$$

where

$$
\phi=\frac{1}{2 \beta} \sqrt{1+4 \beta\left[s+\frac{3 \eta}{\kappa \gamma}\left(1-\frac{1}{1-\gamma+\gamma \sqrt{\kappa s} \operatorname{coth} \sqrt{\kappa s}}\right)\right]}
$$

Substituting Eq.(21) into the following theorem (note that $\int_{0}^{\infty} C_{e} d \tau=1$ ):

$$
\left(\begin{array}{l}
\begin{array}{l}
\text { nth absolute } \\
\text { moment of } \\
\text { output } \\
\text { signal }
\end{array}
\end{array}\right)=\mu_{n}=\frac{\int_{0}^{\infty} C_{e} \tau^{n} d \tau}{\int_{0}^{\infty} C_{e} d \tau}=(-1)^{n}\left(\frac{d^{n} \bar{C}_{e}}{d s^{n}}\right)_{s=0}
$$

one obtains

$$
\begin{aligned}
& \mu_{1}=(1+\eta) f \\
& \mu_{2}=\frac{2}{3} \kappa \eta\left(\gamma+\frac{1}{5}\right) f+[2(1+3 \beta) f-3](1+\eta)^{2}+2 \mu_{1}^{2}
\end{aligned}
$$

where

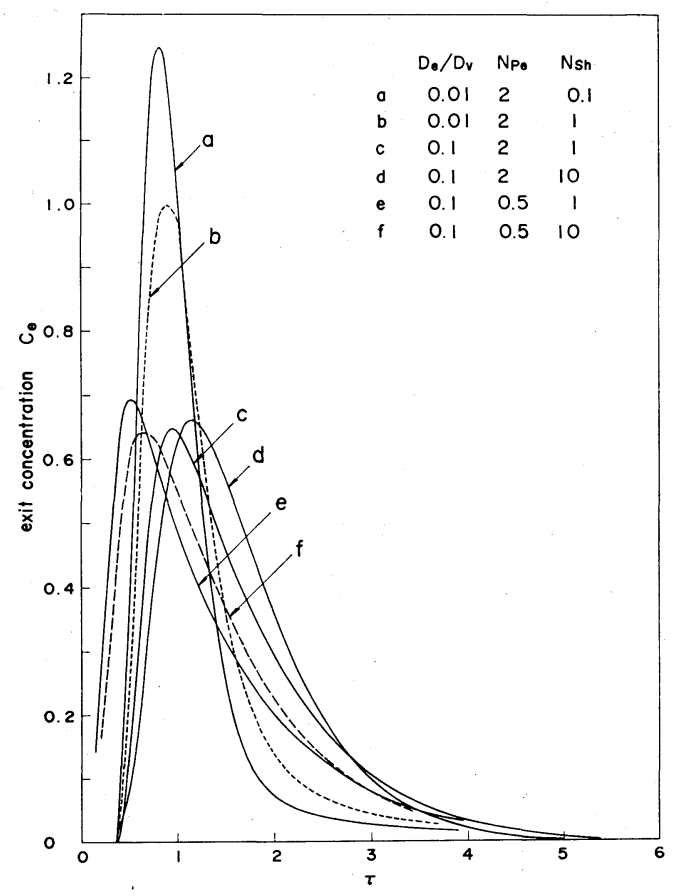

Fig. 1 Delta responses calculated by Eq.(15), with assumption of $\varepsilon_{p}=0.5, \varepsilon_{b}=0.4, N_{S c} N_{R e}=10$ and $L / D_{p}=\mathbf{1 0}$

$$
f=1-\beta+\beta e^{-1 / \beta}
$$

Note that when $L / D_{p}$ is so large that $\beta$ is sufficiently small, Eqs.(24) and (25), respectively, reduce to

$$
\begin{aligned}
\mu_{1} & =(1+\eta)(1-\beta) \\
& \fallingdotseq(1+\eta) \\
\mu_{2} & =\frac{2}{3} \kappa \eta\left(\gamma+\frac{1}{5}\right)(1-\beta)+\left(1-4 \beta^{2}\right)(1+\eta)^{2} \\
& \fallingdotseq \frac{2}{3} \kappa \eta\left(\gamma+\frac{1}{5}\right)+(1+\eta)^{2}
\end{aligned}
$$

second central moment $=\mu_{2}-\mu_{1}^{2}$

$$
\begin{aligned}
& =\frac{2}{3} \kappa \eta\left(\gamma+\frac{1}{5}\right)(1-\beta)+2 \beta\left(1-\frac{5}{2} \beta\right)(1+\eta)^{2} \\
& \fallingdotseq \frac{2}{3} \kappa \eta\left(\gamma+\frac{1}{5}\right)+2 \beta(1+\eta)^{2}
\end{aligned}
$$

where Eqs. $\left(24^{\prime \prime}\right),\left(25^{\prime \prime}\right)$ and $\left(25 \mathrm{~A}^{\prime \prime}\right)$ are identical with those derived earlier by Kubin ${ }^{8)}$ and Kucera ${ }^{9)}$ under the assumption of infinite bed length.

The error of approximating Eqs. $\left(24^{\prime}\right),\left(25^{\prime}\right)$ and $\left(25 \mathrm{~A}^{\prime}\right)$, respectively, to Eqs. $\left(24^{\prime \prime}\right),\left(25^{\prime \prime}\right)$ and $\left(25 \mathrm{~A}^{\prime \prime}\right)$ depends on the value of $\beta$.

When Eqs. $\left(24^{\prime \prime}\right)$ and $\left(25 \mathrm{~A}^{\prime \prime}\right)$ are used for parameter estimation, as was done by Smith et al..$^{4,11,12)}$, the maximum error caused is considered to be the approximation of $1-2.5 \beta \fallingdotseq 1$. If this error is expected to be within 5 per cent, it should be that $\beta \leq 0.02$. For beds of fine particles Smith et al. have obtained low $N_{P e}$ values, but their $L / D_{p}$ is sufficiently large so that the approximation of infinite bed length is sufficiently satisfied. For beds with $N_{P e}=2$, as is expected for beds 


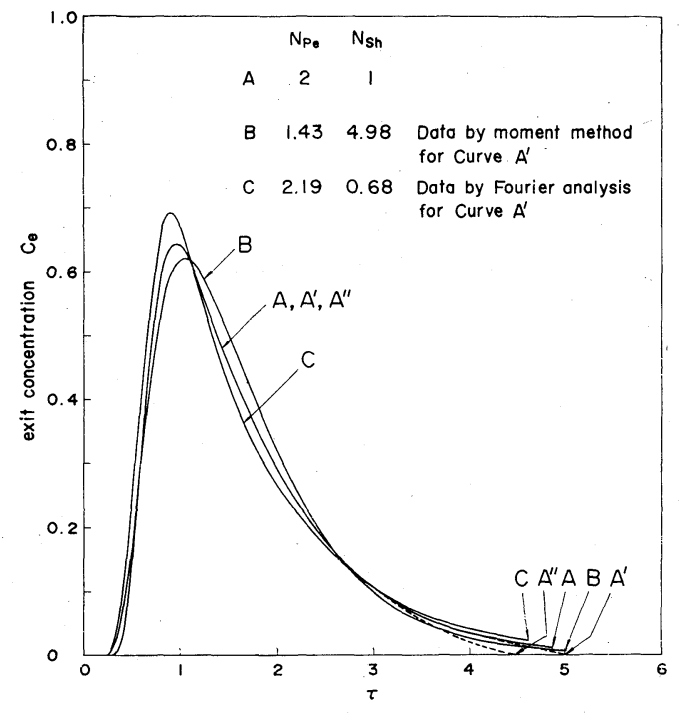

Fig. 2 Comparison of original outputs (Gurves $A^{\prime}$ and $A^{\prime \prime}$ ) and the predicted outputs resulting from moment method and Fourier analysis; $\boldsymbol{\varepsilon}_{p}=\mathbf{0 . 5}, \quad \boldsymbol{\varepsilon}_{b}=\mathbf{0 . 4}, \quad N_{S c} N_{R e}=10, \quad L / D_{p}=10$ and $D_{e} / D_{v}=0.1$ are assumed.

of relatively large particles in the turbulent flow region, the condition of $\beta \leq 0.02$ is satisfied when $L / D_{p} \geq 25$.

\section{Delta Response in Fourier Series}

Substitution of $s=i \omega$ into Eq.(21) gives

$$
\bar{C}_{e}(i \omega)=\operatorname{Re}\left(\bar{C}_{e}\right)+i \operatorname{Im}\left(\bar{C}_{e}\right)
$$

where $\operatorname{Re}\left(\overline{C_{e}}\right)$ and $\operatorname{Im}\left(\overline{C_{e}}\right)$ are shown in Appendix B.

In the following Fourier series of $C_{e}$ in the range of $0<\tau<T$

$$
C_{e}=2 b_{0}+\sum_{n=1}^{\infty}\left(a_{n} \sin \frac{n \pi \tau}{T}+b_{n} \cos \frac{n \pi \tau}{T}\right)
$$

the Fourier coefficients are related to $\bar{G}_{e}(i n \pi / T)$ as follows ${ }^{6)}$ :

$$
\begin{aligned}
a_{n} & =\frac{1}{T} \int_{0}^{2 T} C_{e} \sin \frac{n \pi \tau}{T} d \tau \\
& =-\frac{1}{T} \operatorname{Im}\left(\overline{C_{e}}\left(i \frac{n \pi}{T}\right)\right) \\
b_{n} & =\frac{1}{T} \int_{0}^{2 T} C_{e} \cos \frac{n \pi \tau}{T} d \tau \\
& =\frac{1}{T} \operatorname{Re}\left(\bar{C}_{e}\left(i \frac{n \pi}{T}\right)\right)
\end{aligned}
$$

\section{Comparison of Moment Method and Fourier Analysis}

Curve A on Fig. 2 is a theoretical peak with the following values:

\begin{tabular}{|c|c|c|c|c|c|}
\hline & \multicolumn{2}{|c|}{ Curve $A^{\prime}$} & \multicolumn{2}{|c|}{ Curve $A^{\prime \prime}$} \\
\hline & & $N_{P e}$ & $N_{S h}$ & $N_{P e}$ & $N_{S t}$ \\
\hline \multicolumn{2}{|c|}{ Moment method (a) } & 1.43 & 4.98 & 1.09 & -2.54 \\
\hline Fourier & $n=1$ & 2.48 & 0.14 & (c) & (c) \\
\hline \multirow[t]{6}{*}{ analysis (b) } & 2 & 1.86 & 0.47 & 1.55 & 0.36 \\
\hline & 3 & 1.87 & 0.78 & 1.44 & 0.80 \\
\hline & 4 & 1.95 & 0.96 & 1.45 & 1.28 \\
\hline & 5 & 2.15 & 0.96 & 1.50 & 1.66 \\
\hline & 6 & 2.80 & 0.79 & 1.65 & 1.65 \\
\hline & mean & 2.19 & 0.68 & 1.52 & 1.15 \\
\hline
\end{tabular}

$$
\begin{aligned}
& \varepsilon_{p}=0.5, \quad \varepsilon_{b}=0.4, \quad L / D_{p}=10, \quad N_{S c} N_{R e}=10 \\
& D_{e} / D_{v}=0.1, \quad N_{P e}=2, \quad N_{S h}=1
\end{aligned}
$$

Consider the hypothetical output signals, Gurve $\mathrm{A}^{\prime}$ and $\mathrm{A}^{\prime \prime}$, which are identical with Curve A except for
Table 1 Data estimated by moment method and Fourier analysis

(a) $\quad \mu_{1}=1.63$ and $\mu_{2}=3.35$, for Curve $\mathrm{A}^{\prime}$ $\mu_{1}=1.59$ and $\mu_{2}=3.13$, for Curve $\mathrm{A}^{\prime \prime}$

(b) It is assumed that $T=20$ so that $T /$ (the time at which tail vanishes $)=20 / 5=4$ for Curve $A^{\prime}$, and $=20 / 4.5=4.4$ for Curve $A^{\prime \prime}$.

(c) No solution is found.

the tailing portion. The only difference is that Curve $\mathrm{A}^{\prime}$ (zero concentration at $\tau>5$ ) and Curve $\mathrm{A}^{\prime \prime}$ (zero concentration at $\tau>4.5$ ) have a short tail, while Curve $\mathrm{A}$ has a long tail. Note that the tail of Gurve $A$ is

$$
\begin{aligned}
\tau & =4.5 & & 5 \\
C_{e} & =0.018 & & 0.010
\end{aligned}
$$

Since the tail is not accurately measurable, such small misrecording and/or experimental error will be quite frequently incurred:

$$
\begin{gathered}
\frac{\left(C_{e}\right)_{\tau=4.5}}{\text { peak value of } C_{e}}=2.8 \% \\
\frac{\left(C_{e}\right)_{\tau=5}}{\text { peak value of } C_{e}}=1.5 \%
\end{gathered}
$$

Our interest is what parameter values would be determined from these hypothetical outputs. I

Let us assume that all the parameter values except $N_{P e}$ and $N_{S h}$ are known and that the $N_{P e}$ and $N_{S h}$ are to be determined by the moment method ( $N_{P e}$ from Eq.(24) for $\mu_{1}$ and $N_{S h}$ from Eq.(25) for $\mu_{2}$ ) and Fourier analysis (solving simultaneously each set of $a_{n}$ and $b_{n}$ for $N_{P e}$ and $N_{S h}$ ).

The computed data are shown in Table 1. It should be noted that the originally assumed data are $N_{P e}=2$ and $N_{S h}=1$. In the case of Curve $\mathrm{A}^{\prime}$, the Fourier analysis looks better than the moment method as far as the estimated data are concerned, although the predicted output resulting from the Fourier analysis (Curve C, Fig. 2) deviates from Curve $\mathrm{A}^{\prime}$ as much as that from the moment method (Curve B).

From Curve A", which has the shortest tail, the moment method gives negative $N_{S h}$ value. With regard to the Fourier analysis, from $a_{1}$ and $b_{1}$ no solution (for $N_{P e}$ and $\left.N_{S h}\right)$ is found to exist, at least in the range of $N_{S h}=0.1-10$, but the mean $N_{P e}$ and $N_{S h}$ values for $n=2$ to 6 may be regarded as reasonable.

The moment method is much simpler than the Fourier analysis, but the former has a disadvantage in that the higher moments are seriously affected by error 
in the tail. The Fourier analysis is not so sensitive to error in the tail, but it also has a disadvantage, in that the estimated data depend on $n$ and the period $T$.

Finally, it is concluded that Fourier analysis may be more safely applied than the moment method to signals of which the tail is not accurately measured.

\section{Appendix A}

Similarly to Amundson ${ }^{1)}$, the following change of variables converts the system of Eqs. (8) to (14) to a form in which a finite Fourier transform can be applied:

$$
W=C e^{-\frac{x}{2 \beta}}, w=c_{i} e^{-\frac{x}{2 \beta}}
$$

Then Eqs.(8) to (14) become

$$
\begin{aligned}
\frac{\partial W}{\partial \tau}= & \beta \frac{\partial^{2} W}{\partial x^{2}}-\frac{1}{4 \beta} W-\frac{3 \eta}{\kappa}\left(\frac{\partial w}{\partial \rho}\right)_{\rho=1} \\
\kappa \frac{\partial w}{\partial \tau}= & \frac{\partial^{2} w}{\partial \rho^{2}}+\frac{2}{\rho} \frac{\partial w}{\partial \rho} \\
& W(x, 0)=0 \\
& w(\rho, x, 0)=0 \\
& \gamma\left(\frac{\partial w}{\partial \rho}\right)_{\rho=1}=W-(w)_{\rho=1} \\
& W(0, \tau)=\delta(\tau=0) \\
& \left(\frac{\partial W}{\partial \mathrm{x}}\right)_{x=1}+\frac{1}{2 \beta}(W)_{x=1}=0
\end{aligned}
$$

When the following Fourier transform is applied:

$$
G_{n}=\int_{0}^{1} W \sin \alpha_{n} x d x=G
$$

the inverse transform is given by

$$
W=2 \sum_{n=1}^{\infty} \frac{\sin \alpha_{n} x}{1-\frac{\sin 2 \alpha_{n}}{2 \alpha_{n}}} G
$$

with similar expressions for $w$.

Applying the transform, Eqs.(A1) to (A7) become

$$
\begin{gathered}
\frac{\partial G}{\partial \tau}=-\left(\frac{1}{4 \beta}+\beta \alpha_{n}^{2}\right) G+\beta \alpha_{n} \delta(\tau=0)-\frac{3 \eta}{\kappa}\left(\frac{\partial g}{\partial \rho}\right)_{\rho=1} \\
\kappa \frac{\partial g}{\partial \tau}=\frac{\partial^{2} g}{\partial \rho^{2}}+\frac{2}{\rho} \frac{\partial g}{\partial \rho} \\
\quad G=0, \text { at } \tau=0 \\
g=0, \text { at } \tau=0 \\
\gamma\left(\frac{\partial g}{\partial \rho}\right)_{\rho=1}=G-(g)_{\rho=1}
\end{gathered}
$$

where $\alpha_{n}$ is a root of

$$
\tan \alpha_{n}=-2 \beta \alpha_{n}
$$

When the Laplace transform is applied to Eqs.(A10) to (A14), the solutions are easily obtained as

$$
\begin{aligned}
& g=\beta \alpha_{n} \sum_{j=1}^{\infty} \frac{\sinh \left(\rho \sqrt{\kappa s_{j}}\right)}{\rho\left(\frac{d Q_{n}}{d s}\right)_{s_{j}}} e^{s_{j} \tau} \\
& G=\beta \alpha_{n} \sum_{j=1}^{\infty} \frac{(1-\gamma) \sinh \sqrt{\kappa s_{j}}+\gamma \sqrt{\kappa s_{j}} \cosh \sqrt{\kappa s_{j}}}{\left(\frac{d Q_{n}}{d s}\right)_{s_{j}}} e^{s_{j} \tau}
\end{aligned}
$$

where $s_{j}$ is a root of

$$
Q_{n}\left(s_{j}\right)=\left[(1-\gamma)\left(\frac{1}{4 \beta}+\beta \alpha_{n}^{2}+s_{j}\right)-\frac{3 \eta}{\kappa}\right] \sinh \sqrt{\kappa s_{j}}
$$

$$
+\left[\gamma\left(\frac{1}{4 \beta}+\beta \alpha_{n}^{2}+s_{j}\right)+\frac{3 \eta}{\kappa}\right] \sqrt{\kappa s_{j}} \cosh \sqrt{\kappa s_{j}}=0
$$

The bulk concentration is given by

$$
C=2 e^{\frac{x}{2 \beta}} \sum_{n=1}^{\infty} \frac{\sin \alpha_{n} x}{1-\frac{\sin 2 \alpha_{n}}{2 \alpha_{n}}} G
$$

Substituting $\sqrt{\kappa s_{j}}=i z_{j}$,

$$
\begin{aligned}
C & =2 \beta e^{\frac{x}{2 \beta}} \sum_{n=1}^{\infty} \sum_{j=1}^{\infty} \frac{\alpha_{n} \sin \alpha_{n} x}{1-\frac{\sin 2 \alpha_{n}}{2 \alpha_{n}}} \\
& \times \frac{(1-\gamma) \sin z_{j}+\gamma z_{j} \cos z_{j}}{A_{n, j} \sin z_{j}+B_{n, j} \cos z_{j}} e^{\left(-z_{j}^{2} z / \kappa\right)}
\end{aligned}
$$

where $A_{n, j}$ and $B_{n, j}$ are defined in the text.

Note that when the bed may be assumed to be of infinite length, the following boundary condition is used

$$
C=0 \text {, at } X=\infty
$$

Only major modification is to apply a Fourier integral instead of using Eqs.(A8) and (A9). The solution is

$$
C_{e}=\frac{2 \beta D_{p}}{\pi L} \int_{0}^{\infty} \sum_{j=1}^{\infty} \alpha \sin \alpha d \alpha \frac{(1-\gamma) \sin z_{j}+\gamma z_{j} \cos z_{j}}{A_{j} \sin z_{j}+B_{j} \cos z_{j}} e^{-\left(2 \beta z_{j}^{2} \tau^{-\kappa}\right) /(2 \kappa \beta)}
$$

where $A_{j}, B_{j}$ and $z_{j}$ are, respectively, given by Eqs.(16), (17) and (19) provided that $\alpha_{n}$ is replaced by $\alpha$.

\section{Appendix B}

Substitution of $s=i w$ into Eq.(21) gives

$$
\begin{aligned}
& \bar{C} e(i w)=\operatorname{Re}(\bar{C} e)+i \operatorname{Im}(\bar{C} e) \\
& \operatorname{Re}(\bar{C} e)=e^{1 / 2 \beta}\left[e^{b_{c}}\left\{M \cos b_{s}-N \sin b_{s}\right\}\right. \\
& \left.+e^{-b_{c}}\left\{(1-M) \cos b_{s}-N \sin b_{s}\right\}\right] \\
& \operatorname{Im}(\bar{C} e)=e^{1 / 2 \beta}\left[e^{b_{c}}\left\{N \cos b_{s}+M \sin b_{s}\right\}-e^{-b_{c}}\right. \\
& \left.\left\{N \cos b_{s}+(1-M) \sin b_{s}\right\}\right] \\
& \text { where } b_{c}=\left(\zeta^{2}+\phi^{2}\right)^{1 / 4} \cos \left(\frac{1}{2} \tan ^{-1} \frac{\phi}{\zeta}\right) \\
& b_{s}=\left(\zeta^{2}+\phi^{2}\right)^{1 / 4} \sin \left(\frac{1}{2} \tan ^{-1} \frac{\phi}{\zeta}\right) \\
& \zeta=\left(\frac{1}{2 \beta}\right)^{2}+\frac{3 \eta}{\kappa \gamma \beta}\left[1-\frac{l \cos \theta \sinh \theta+m \sin \theta \cosh \theta}{l^{2}+m^{2}}\right] \\
& \phi=\frac{\omega}{\beta}-\frac{3 \eta}{\kappa \gamma \beta}\left[\frac{l \sin \theta \cosh \theta-m \cos \theta \sinh \theta}{l^{2}+m^{2}}\right] \\
& l=\gamma \theta(\cos \theta \cosh \theta-\sin \theta \sinh \theta)+(1-\gamma) \cos \theta \sinh \theta \\
& m=r \theta(\cos \theta \cosh \theta+\sin \theta \sinh \theta)+(1-\gamma) \sin \theta \cosh \theta \\
& \theta=\sqrt{\frac{\kappa \omega}{2}} \\
& M=-\frac{F(D-F)+G(E-G)}{(D-F)^{2}+(E-G)^{2}} \\
& N=\frac{F(E-G)-G(D-F)}{(D-F)^{2}+(E-G)^{2}} \\
& D=\left[\left(\frac{1}{2 \beta}+b_{c}\right) \cos b_{s}-b_{s} \sin b_{s}\right] e^{(1 / 2 \beta)+b_{c}} \\
& E=\left[\left(\frac{1}{2 \beta}+b_{c}\right) \sin b_{s}+b_{s} \cos b_{s}\right] e^{(1 / 2 \beta)+b_{c}} \\
& F=\left[\left(\frac{1}{2 \beta}-b_{c}\right) \cos b_{s}-b_{s} \sin b_{s}\right] e^{(1 / 2 \beta)-b_{c}} \\
& G=\left[\left(\frac{1}{2 \beta}-b_{c}\right) \sin b_{s}+b_{s} \cos b_{s}\right] e^{(1 / 2 \beta)-b_{c}}
\end{aligned}
$$




\section{Nomenclature}

$C=$ dimensionless tracer concentration in bulk stream; (concentration)/(amount of tracer input/extraparticle void volume of bed)

$\begin{array}{ll}C_{e} & =C \text { at reactor exit } \\ \bar{C}_{e} & =\text { Laplace transform of } C_{e}\end{array}$

$c_{i}=$ dimensionless tracer concentration in particle; (concentration)/(amount of tracer input/extraparticle void volume of bed)

$D_{a x} \quad=$ axial fluid mixing coefficient based on extraparticle void area

$D_{e} \quad=$ effective diffusivity in porous solid

$D_{p} \quad=$ particle diameter

$D_{v} \quad=$ molecular diffusivity

$k_{f} \quad=$ particle-fluid mass transfer coefficient

$L=$ packed-bed height

$N_{P e}=\frac{D_{p} U}{D_{a x}}$

$N_{R e}=\frac{D_{p \varepsilon_{b}} U}{\nu}$

$N_{S c} \quad=\frac{\nu}{D_{v}}$

$N_{S h}=\frac{k_{f} D_{p}}{D_{v}}$

$R \quad=$ particle radius

$r \quad=\quad$ radial distance variable

$t \quad=$ time

$U \quad=$ interstitial fluid velocity

$X \quad=$ axial distance variable

$x \quad=\frac{X}{L}$

$z_{j} \quad=$ root of Eq.(19)

$\alpha_{n} \quad=$ root of Eq.(18)

$\beta=\frac{1}{N_{P e}\left(\frac{L}{D_{p}}\right)}$ $\left[\mathrm{cm}^{2} / \mathrm{sec}\right]$

$\left[\mathrm{cm}^{2} / \mathrm{sec}\right]$

$[\mathrm{cm}]$

$\left[\mathrm{cm}^{2} / \mathrm{sec}\right]$

$[\mathrm{cm} / \mathrm{sec}]$

[cm]

$[-]$

$[-]$

$[-]$

$[-]$

[cm]

[cm]

[sec]

$[\mathrm{cm} / \mathrm{sec}]$

[cm]

$[-]$

$[-]$

$[-]$

$[-]$

$$
\gamma=\frac{2\left(\frac{D_{e}}{D_{v}}\right)}{N_{S h}}
$$

$\delta=$ Kronecker's delta function

$\varepsilon_{b} \quad=$ extraparticle void fraction

$\varepsilon_{p} \quad=$ intraparticle void fraction

$\eta \quad=\varepsilon_{p}$

$\kappa=\frac{N_{R e} N_{S c}\left(\frac{\varepsilon_{p}}{\varepsilon_{b}}\right)}{4\left(\frac{D_{e}}{D_{v}}\right)\left(\frac{L}{D_{p}}\right)}$

$\mu_{n} \quad=n$-th absolute moment

$\nu=$ kinematic viscosity

$\rho \quad=\frac{r}{R}$

\section{Literature Gited}

1) Amundson, N. R.: Ind. Eng. Chem., 48, 26 (1956)

2) Anderssen, A. S. and E. T. White: Chem. Eng. Sci., 25, 1015 (1970)

3) Böxkes, W. and H. Hofmann: Chem. Ing. Tech., 44, 882 (1972)

4) Gerro, R. L. and J. M. Smith: AIChE J., 16, 1034 (1970)

5) Clements, W. C.: Chem. Eng. Sci., 24, 957 (1969)

6) Gangwal, S. K., R. R. Hudgins, A. W. Bryson and P. L. Silveston: Can. J. Chem. Eng., 49, 113 (1971)

7) Hopkins, M. J., A. J. Sheppard and P. Eisenklam: Chem. Eng. Sci., 24, 1131 (1969)

8) Kubin, M.: Collection Czechoslov. Chem. Commun., 30, 1104 (1965); 2900 (1965)

9) Kucera, E.: J. Chromatography, 19, 237 (1965)

10) Ostergaard, K. and M. L. Michelsen: Can. J. Chem. Eng., 47, 107 (1969)

11) Schneider, P. and J. M. Smith: AIChE J., 14, 762 (1968)

12) Suzuki, M. and J. M. Smith: Chem. Eng. J., 3, 256 (1972) 